\title{
Prevalencia de tumores apendiculares en pacientes operados de apendicectomía en el Hospital General de México "Dr. Eduardo Liceaga”, revisión a 10 años
}

\author{
Prevalence of appendiceal tumors in patients who underwent appendectomy \\ in the Hospital General de México "Dr. Eduardo Liceaga", a 10-year review
}

Sinuhé Álvarez-Álvarez, ${ }^{*}$ Luis Gabriel González-Pérez, ${ }^{*}$ Eder Alejandro Sánchez-Pérez, ${ }^{*}$ Marco Antonio Madrigal-Téllez, ${ }^{*}$ Luis Mauricio Hurtado-López*

Palabras clave: Apéndice, tumor, apendicitis, cáncer apendicular.

Key words: Appendix, tumor, appendicitis, appendix cancer.

\footnotetext{
* Servicio de Cirugía General, Hospital General de México "Dr. Eduardo Liceaga", Ciudad de México, México.
}

\section{RESUMEN}

Introducción: Dentro de las enfermedades que afectan al apéndice se encuentran las neoplasias, con diferente comportamiento según su histología. Este estudio tiene como objetivo determinar la prevalencia de los tumores apendiculares incidentales, su frecuencia por tipo histológico, así como su distribución por edad y sexo en el Hospital General de México "Dr. Eduardo Liceaga". Material y métodos: Estudio de cohorte en el cual se revisaron los reportes de piezas de apéndice cecal en un periodo de 10 años; se evaluaron las siguientes variables: sexo, edad, diagnóstico que motivó la cirugía y diagnóstico histopatológico. Resultados: De 6,242 reportes de apéndice cecal, 1.68\% correspondieron a neoplasias, $90.47 \%$ fueron benignas (neuroma y adenoma) y $9.53 \%$ malignas (adenocarcinoma y carcinoide). De las neoplasias malignas, el carcinoide correspondió al $60 \%$, con predominio en mujeres $(5: 1)$ y edad promedio de 25 años ( $\mathrm{DE} \pm 8.78)$. El adenocarcinoma correspondió al $20 \%$, siendo todas mujeres con edad promedio de 36 años $(\mathrm{DE} \pm 2.12)$. El adenocarcinoma mucinoso representó el $20 \%$, sin preferencia por sexo, con edad promedio de 47 años (DE \pm 13.43$)$. La edad de presentación para neoplasias benignas fue de 47.9 años en promedio, y para malignas, de 32.1 años $(\mathrm{p}=0.001)$. De las neoplasias benignas, el $72 \%$ se presentó en mujeres, y de las neoplasias malignas, $80 \%(\mathrm{p}=0.900)$. Conclusiones: $\mathrm{La}$ prevalencia de los tumores apendiculares incidentales fue de $1.68 \%$, para neoplasia benigna fue de $1.52 \%$ y maligna, de $0.16 \%$. De las neoplasias benignas, la más frecuente fue neuroma apendicular $(89.47 \%)$, seguida de adenoma mucinoso $(9.47 \%)$ y adenoma seroso $(1.05 \%)$. Las malignas consistieron en tumor carcinoide $(60 \%)$, adenocarcinoma $(20 \%)$ y adenocarcinoma mucinoso $(20 \%)$. La edad de presentación para neoplasias malignas fue la cuarta década de vida y para benignas, la quinta.

\section{ABSTRACT}

Introduction: Appendix tumors represent a fraction of the appendicular pathology, with different behavior depending on their histological pattern. This study has the objective to determine the prevalence of incidental appendiceal tumors, their frequency by histological type and distribution by age and sex at the Hospital General de México "Dr. Eduardo Liceaga". Material and methods: A cohort study in which cecal appendix histological reports were checked in a period of 10 years, evaluating the following variables: sex, age, the diagnosis that determined surgery and histological result. Results: Of 6,242 reports of cecal appendix, 1.68\% corresponded to neoplasm. Of these, $90.47 \%$ were benign (neuroma and adenoma) and $9.53 \%$ malignant (adenocarcinoma and carcinoid tumor). Of malignant neoplasms, the carcinoma represented $60 \%$, with a predominance in women (5:1) and an average age of 25 years ( $S D \pm 8.78$ ). Adenocarcinoma represented $20 \%$; all of them occurred in women with an average age of 36 years ( $S D \pm 2.12)$. Mucinous adenocarcinoma represented $20 \%$, with no sex preference and an average age of 47 years $(S D \pm 13.43)$. The age of onset for benign tumors was, on average, 47.9 years, and 32.1 years for malignant tumors $(p=0.001)$. Of benign neoplasms, $72 \%$ occurred in women, and $80 \%$ of the malignant ones $(p=0.900)$. Conclusions: The prevalence of incidental appendiceal tumors was $1.68 \%$; for benign neoplasms, $1.52 \%$, and $0.16 \%$ for malignant. The most common benign neoplasm was appendix neuroma (89.47\%), followed by mucinous adenoma (9.47\%), and serous adenoma $(1.05 \%)$. Malignant neoplasms were carcinoid tumor (60\%), adenocarcinoma (20\%), and mucinous adenocarcinoma (20\%). The age of onset for malignancies was the fourth decade of life, and for benign tumors, the fifth. 


\section{INTRODUCCIÓN}

$\mathrm{L}$ os tumores apendiculares constituyen un grupo heterogéneo de neoplasias; representan sólo una parte de las enfermedades apendiculares, en donde predominan los procesos de origen inflamatorio. ${ }^{1,2}$ La OMS divide a los tumores del apéndice cecal en dos grupos: epiteliales y no epiteliales; en el primer grupo se incluye al adenoma, carcinoma, tumor carcinoide (tumor neuroendocrino bien diferenciado), carcinoide tubular, carcinoide mucinoso y mixto (carcinoide-adenocarcinoma); dentro de los tumores no epiteliales se incluyen el neuroma, lipoma, leiomioma, tumores del estroma gastrointestinal, leiomiosarcoma y sarcoma de Kaposi, entre otros. ${ }^{3,4}$

El estudio anatomopatológico de rutina del apéndice cecal puede encontrar anomalías no sospechadas en alrededor del 5\% de los casos, siendo los tumores apendiculares parte importante de estos hallazgos. ${ }^{5}$

Típicamente se describen los tumores del apéndice como benignos o malignos, teniendo estos últimos diferente comportamiento según su tipo histológico, tamaño, localización y grado de diferenciación; los más frecuentes son el carcinoma y el adenocarcinoma. ${ }^{6}$

Los tumores carcinoides en el apéndice fueron descritos por Beger en 1882; en 1907 se acuñó el término "carcinoide" para describir tumores similares al adenocarcinoma, pero de comportamiento menos agresivo,, 8 con comportamiento neuroendocrino. ${ }^{9,10}$ Suelen encontrarse en el tracto gastrointestinal $(74 \%),{ }^{8}$ pero pueden aparecer en otras localizaciones como páncreas u ovario. ${ }^{11,12} \mathrm{El}$ síndrome carcinoide vinculado a esta neoplasia se asocia a pacientes con metástasis hepáticas y representa menos del $2 \%$ de los casos. 7,9

Del carcinoma apendicular se distinguen cinco tipos: adenocarcinoma, adenocarcinoma mucinoso, carcinoma de células en anillo de sello, carcinoma de células pequeñas y carcinoma indiferenciado. ${ }^{4}$ Con base en su extensión, se pueden dividir en invasivos o no invasivos, dependiendo de si se extienden más allá de la mucosa. ${ }^{13,14}$

De los tumores mucinosos del apéndice, generalmente benignos, se distinguen cuatro tipos: mucocele simple, hiperplasia mucosa
(5-25\%), adenoma mucinoso (63-84\%) y adenocarcinoma mucinoso (1\%). ${ }^{15}$

En cuanto a los tumores benignos, el más frecuente es el neuroma apendicular, ${ }^{3,4}$ que se caracteriza por presentar una base de colágeno con una gran variedad de células, que incluyen fibras nerviosas, células espinosas, proteínas, células endocrinas y eosinófilos. ${ }^{4}$ Se cree que procesos inflamatorios subclínicos del apéndice provocan proliferación del tejido neural, y se sugiere el término "apendicopatía neurogénica" para los pacientes con síntomas crónicos de apendicitis sin otro hallazgo más que neuroma apendicular y que mejoran tras la cirugía, aunque este término aún no es del todo aceptado por patólogos y cirujanos. ${ }^{16,17}$

Si bien existen series de Latinoamérica que estiman la prevalencia de los tumores apendiculares en $0.8 \%^{2}$ y series mexicanas con una prevalencia del $0.58 \%,{ }^{1}$ en la actualidad se desconoce la prevalencia de esta enfermedad en la población del Hospital General de México, así como los factores epidemiológicos que la acompañan; es importante conocer estas cifras para valorar si nuestra actual forma de actuar es correcta o si se deben implementar medidas encaminadas a la prevención, detección y manejo de este padecimiento, ya que aunque estos tumores raramente son diagnosticados antes o durante la cirugía, cuando se sospecha de su diagnóstico intraoperatorio, puede no estar clara la manera correcta de actuar del cirujano.

El presente estudio tiene como objetivo determinar la prevalencia de los tumores apendiculares incidentales, su frecuencia por tipo histológico, así como su distribución por edad y sexo en el Hospital General de México "Dr. Eduardo Liceaga".

\section{MATERIAL Y MÉTODOS}

Se trata de un estudio de cohorte en el cual se revisaron los registros de piezas de apéndice cecal de un periodo de 10 años, correspondiente de enero de 2004 a diciembre de 2013, del Departamento de Patología del Hospital General de México "Dr. Eduardo Liceaga". Se clasificaron las piezas con base en su diagnóstico histopatológico y se observó su distribución por edad y sexo. Los resultados fueron analizados utilizando el programa Microsoft ${ }^{\circledR}$ Excel 2013. 


\section{RESULTADOS}

Se encontraron 6,222 registros de piezas de apéndice cecal, los cuales se clasificaron con base en sus hallazgos, tal como se muestra en el cuadro $I$.

Hubo un total de 105 pacientes con diagnóstico de tumores apendiculares, que se clasificaron en benignos y malignos: se obtuvieron 10 casos de tumores malignos $(9.52 \%)$ y 95 de tumores benignos $(90.47 \%)$.

Cuadro I. Hallazgos del total de piezas de apéndice cecal en un periodo de 10 años.

\begin{tabular}{lrc} 
Hallazgos & \multicolumn{1}{c}{$\mathbf{n}$} & \multicolumn{1}{c}{$\%$} \\
\hline Tuberculosis & 1 & 0.016 \\
Apéndice normal & 414 & 6.95 \\
Hiperplasia folicular & 599 & 9.59 \\
Apendicitis sin perforación & 3,103 & 49.71 \\
Apendicitis con perforación & 2,000 & 32.04 \\
Tumor apendicular & 105 & 1.68
\end{tabular}

Cuadro II. Características de los pacientes con tumor benigno apendicular.

\begin{tabular}{|c|c|c|c|}
\hline \multirow[b]{2}{*}{ Tipo de tumor } & \multicolumn{2}{|c|}{ Número de casos por sexo } & \multirow{2}{*}{$\begin{array}{l}\text { Edad promedio en años } \\
\qquad( \pm \mathrm{DE})\end{array}$} \\
\hline & Masculino & Femenino & \\
\hline Neuroma apendicular & $23(27 \%)$ & $62(73 \%)$ & $47.14( \pm 19.65)$ \\
\hline Adenoma mucinoso & $3(33 \%)$ & $6(66 \%)$ & $54.8( \pm 28.57)$ \\
\hline Adenoma seroso & $0(0 \%)$ & $1(100 \%)$ & 59 \\
\hline
\end{tabular}

\begin{tabular}{|c|c|c|c|}
\hline \multicolumn{4}{|c|}{$\begin{array}{l}\text { Cuadro III. Características de los } \\
\text { pacientes con tumor maligno apendicular. }\end{array}$} \\
\hline \multirow[b]{2}{*}{ Tipo de tumor } & \multicolumn{2}{|c|}{ Número de casos por sexo } & \multirow{2}{*}{$\begin{array}{c}\text { Edad promedio en años } \\
( \pm \mathrm{DE})\end{array}$} \\
\hline & Masculino & Femenino & \\
\hline Tumor carcinoide & $1(17 \%)$ & $5(83 \%)$ & $25.5( \pm 8.78)$ \\
\hline Adenocarcinoma & $0(0 \%)$ & $2(100 \%)$ & $36.5( \pm 2.12)$ \\
\hline $\begin{array}{l}\text { Adenocarcinoma } \\
\text { mucinoso }\end{array}$ & $1(50 \%)$ & $1(50 \%)$ & $47.5( \pm 13.43)$ \\
\hline
\end{tabular}

De los 95 individuos con diagnóstico de tumor benigno, 69 fueron del sexo femenino (72\%), con una edad promedio de 47 años (rango de 13 a 91 años); se obtuvieron 85 casos de neuroma apendicular (89.47\%), nueve casos de adenoma mucinoso $(9.47 \%$ ) y un caso de adenoma seroso $(1.05 \%)$. Los detalles de estos sujetos se especifican en el cuadro II.

De las 10 personas con diagnóstico de tumor maligno, ocho fueron del sexo femenino $(80 \%)$ y dos del sexo masculino (20\%), con una edad promedio de 32 años ( $\mathrm{DE} \pm 12.22$ años, rango de 16 a 57 años); se obtuvieron seis casos de carcinoide apendicular (60\%), dos de adenocarcinoma $(20 \%)$ y dos de adenocarcinoma mucinoso (20\%). Los detalles se resumen en el cuadro III.

La edad de presentación de las neoplasias benignas fue de 47.9 años en promedio y de 32.1 años para las neoplasias malignas ( $\mathrm{p}=$ 0.001 ). De las neoplasias benignas, el $72 \%$ se presentó en mujeres, mientras que de las neoplasias malignas, el $80 \%$ ocurrió en este $\operatorname{sexo}(p=0.900)$.

Se estadificó a los pacientes con base en la clasificación TNM para carcinoide y adenocarcinoma apoyados en la descripción histopatológica y el reporte de metástasis tanto por patología como en el dictado quirúrgico. Se encontró que el $100 \%$ de los individuos se encontraba en estadios tempranos. La clasificación y estadio de cada uno de los sujetos se resume en el cuadro IV.

El tratamiento quirúrgico recibido por todas las personas con neoplasia maligna fue apendicectomía; en ocho de los casos (80\%) la cirugía se realizó por sospecha clínica de apendicitis y en dos (20\%) se inició la cirugía por motivos ginecológicos (cesárea e histerectomía); se encontraron alteraciones macroscópicas del apéndice cecal, lo que motivó a realizar apendicectomía.

\section{DISCUSIÓN}

La prevalencia de los tumores apendiculares fue mayor a lo reportado en otras series; su distribución por tipo histológico coincide: el más frecuente de los tumores malignos fue el carcinoide y de los benignos, el neuroma; encontramos una prevalencia mayor en mujeres 


\begin{tabular}{lcc}
\multicolumn{2}{c}{$\begin{array}{c}\text { Cuadro IV. Clasificación y } \\
\text { estadio con base en el TNM. }\end{array}$} \\
\hline & $\begin{array}{c}\text { Clasificación } \\
\text { TNM }\end{array}$ & Estadio \\
\hline Diagnóstico & T3NXM0 & IIA \\
\hline Adenocarcinoma & TisNXM0 & 0 \\
Adenocarcinoma & T1NXM0 & 0 \\
Carcinoide apendicular & T1NXM0 & I \\
Carcinoide apendicular & T1NXM0 & I \\
Carcinoide apendicular & T1NXM0 & I \\
Carcinoide apendicular & T1N1M0 & I \\
Carcinoide apendicular & T1NXM0 & I \\
Carcinoide apendicular & T2NXM0 & I \\
Cistoadenocarcinoma & TisNXM0 & 0 \\
mucinoso & & \\
Cistoadenocarcinoma & \\
mucinoso & & \\
\hline T = Tumor, N = Nódulo, M = Metástasis. &
\end{tabular}

para ambos tipos de tumores, con edad de presentación más temprana que lo descrito en otras series. ${ }^{2,3}$

Con respecto al tumor carcinoide, en nuestra población tiene una prevalencia del $0.096 \%$, ligeramente menor que la reportada por Kleanthis y colaboradores, quienes hallaron una incidencia en la población general de 0.1 a $0.9 \% ;^{8}$ por lo tanto, a pesar de ser el tumor maligno más frecuente, la posibilidad de que en nuestro actuar quirúrgico encontremos uno de ellos es extremadamente baja; ocurre mayormente en mujeres jóvenes, al contrario de lo descrito en otras revisiones, donde comunican mayor prevalencia en mujeres de la quinta década de vida. ${ }^{7,13,14}$

El adenocarcinoma y el adenocarcinoma mucinoso fueron el siguiente tipo de neoplasia maligna más frecuente (ambos con una prevalencia de $0.03 \%$ cada uno) después del tumor carcinoide; son neoplasias poco comunes, tal como lo reportan Zenén Rodríguez y su grupo. ${ }^{13}$ Para el adenoma, se encontró una mayor prevalencia en el sexo femenino, contrariamente a lo publicado por Rodríguez y Casaus, ${ }^{14}$ quienes describen este tipo de tumor con predominio del sexo masculino.
Para los tumores mucinosos, encontramos una prevalencia de $0.17 \%$, menor al $0.2-0.3 \%$ reportado en algunas revisiones. ${ }^{18,19}$ De éstos, el $81.81 \%$ correspondió a adenoma mucinoso y $18.18 \%$ a adenocarcinoma mucinoso; para estos últimos no se encontró predominio por sexo a pesar de que se describen como neoplasias de mayor prevalencia en mujeres. ${ }^{15,20,21}$ Sierra-Montenegro y colegas describen al pseudomixoma como una complicación de este tipo de tumores originada de la diseminación peritoneal causada por perforación iatrogénica o espontánea del apéndice; no fue observada en ninguno de los pacientes de este estudio, tal vez porque es una complicación poco frecuente, con una prevalencia de dos casos por cada 100,000 laparotomías. ${ }^{15}$

En cuanto a los tumores no epiteliales, se registró una prevalencia de $1.52 \%$, una cifra mucho más baja que la reportada por Nikita y Norman Machado, quienes describen una prevalencia de $17 \%$ en adultos, coincidiendo en el neuroma como el tumor benigno más frecuente. ${ }^{22}$

De los individuos con tumor maligno, 80\% inició con cuadro clínico de apendicitis, lo que motivó el procedimiento quirúrgico; en el resto, la apendicectomía se realizó por cambios macroscópicos del apéndice al momento de practicar una cirugía ginecológica, sin sospechar en ninguno de los casos que se tratara de un tumor del apéndice, lo cual no es de extrañar, ya que como se reporta, más del $95 \%$ de estos tumores mide menos de un centímetro al momento de la cirugía, por lo que es extremadamente rara su sospecha. ${ }^{8,11}$

Respecto a las metástasis, éstas son uno de los factores pronósticos más importantes, ya que los sujetos con tumores menores de un centímetro y una resección completa tienen una supervivencia del 90 al 100\% a cinco años $;{ }^{8,10}$ se reportan altas tasas de metástasis en pacientes con invasión del mesoapéndice o la grasa periapendicular. ${ }^{7}$ Todos los individuos de nuestra serie se encontraban en etapas clínicas tempranas, por lo que el actuar quirúrgico hubiera sido el mismo, a pesar de que se hubiese logrado el diagnóstico de tumor carcinoide al momento de la cirugía, y ninguno de ellos habría necesitado un abordaje más agresivo. 


\section{CONCLUSIONES}

La prevalencia de los tumores apendiculares incidentales fue de $1.68 \% ; 1.52 \%$ correspondió a neoplasias benignas y $0.16 \%$ a neoplasias malignas. De las neoplasias benignas, la más frecuente fue el neuroma apendicular en un $89.47 \%$, seguido del adenoma mucinoso en $9.47 \%$ y adenoma seroso en $1.05 \%$.

De las neoplasias malignas, la más frecuente fue el tumor carcinoide, que representó el 60\% del total de estas neoplasias, seguido del adenocarcinoma y el adenocarcinoma mucinoso: ambos representaron un $20 \%$ cada uno.

La edad de presentación de las neoplasias malignas fue la cuarta década de vida, mientras que de las benignas fue la quinta.

El 100\% de las neoplasias fue diagnosticado por estudio histopatológico, sin sospechar en ningún caso su presencia antes o durante la cirugía.

\section{REFERENCIAS}

1. Esmer-Sánchez DD, Martínez-Ordaz JL, RománZepeda P, Sánchez-Fernández P, Medina-González E. Tumores apendiculares. Revisión clínico-patológica de 5,307 apendicectomías. Cir Cir. 2004; 72: 375-378.

2. Butte B, García HM, Torres MJ, Salinas FM, Duarte GI, Pinedo MG, et al. Tumores del apéndice cecal. Análisis anatomoclínico y evaluación de la sobrevida alejada. Rev Chil Cir. 2007; 59: 217-222.

3. Segura SJ, Solís GE, González ST. Carcinoideadenocarcinoma mixto de apéndice cecal. Aspectos terminológicos a propósito de un caso. Rev Esp Pat. 2008; 41: 134-137.

4. Hamilton SR, Aaltonen LA. Pathology and genetics of tumours of the digestive system. 4 . $^{\mathrm{a}} \mathrm{ed}$. Lyon, France: IARC Press; 2000. p. 94-102.

5. Paredes ERM, Martínez JM, García RM. Un caso de asociación de mucocele y tumor carcinoide apendicular. Cir Pediatr. 2006; 19: 250-252.

6. Vázquez PJ, Montero RL, Bandera RN, Orlando R, Vallés GM. Tumor carcinoide apendicular, presentación de casos. Rev Cubana Cir [online]. 2008; 47(4).

7. Dall'Igna P, Ferrari A, Luzzatto C, Bisogno G, Casanova $M$, Alaggio R, et al. Carcinoid tumor of the appendix in childhood: the experience of two Italian institutions. J Pediatr Gastroenterol Nutr. 2005; 40: 216-219.
8. Anastasiadis K, Kepertis C, Lampropoulos V, Tsioulas P, Spyridakis A. Carcinoid tumors of the appendix —last decade experience. J Clin Diagn Res. 2014; 8: 1-2.

9. Roggo A, Wood W, Ottinger L. Carcinoid tumors of the appendix. Ann Surg. 1993; 217: 385-390.

10. Florian MC, Uribe A, Cardona CP. Tumor carcinoide del apéndice cecal. Rev Colomb Cir. 2011; 26: 62-66.

11. Uherek PF, Barría AC, Larraín TC, Birrer GE. Carcinoide apendicular. Comunicación de 6 casos y actualización del tema. Cuad Cir. 2004; 18: 52-56.

12. San-Vicente B, Bardají C, Rigol S, Obiols P, Melo M, Bella R. Estudio retrospectivo del tumor carcinoide apendicular en niños. Cir Pediatr. 2009; 22: 97-99.

13. Rodríguez FZ, Casaus PA, Matos TM. Adenocarcinoma primario del apéndice vermiforme. Rev Cubana Cir [online]. 2010; 49(3).

14. Revuelta PLA, Torres AL, Sánchez SA. Adenocarcinoma primario del apéndice vermiforme. Presentación de un caso. Revista Finlay. 2014; 4: 297-300.

15. Sierra-Montenegro E, Sierra-Luzuriaga G, Leone-Stay G, Quiñonez-Auria C, Salazar-Menéndez V. Cistoadenoma mucinoso de apéndice. Informe de un caso. Cir Cir. 2010; 78: 257-260

16. Kirti G, Anjali S, Vasishta RV. Appendiceal neuroma: report of an elusive neuroma. Trop Gastroenterol. 2011; 32: 332-333.

17. Parteck LI, Thiele A, Scmith-Wankel F, Kessler W, Wondy M, Dombeowsky F, et al. Appendicopathy, a clinical and diagnostic dilemma. Int J Colorectal Dis. 2013; 28: 1081-1089.

18. Zarrelli FR, Rodríguez I, Torres EMA. Tumor carcinoide de apéndice. Revisión de la literatura a propósito de un caso. Acta Científica Estudiantil. 2010; 8: 78-82.

19. Aragón QC, Fierro MR, Rodríguez ZA. Cistadenoma mucinoso del apéndice: reporte de caso. Rev Med MD. 2014; 5: 160-163.

20. Malya FU, Hasbahceci M, Karatepe O, Kocakoc E, Muslumanoglu M. Appendicecal mucocele: clinical and imaging features of 14 cases. Chirurgia (Bucur). 2014; 109: 788-793.

21. Badilla MJ, Cambronero AN. Mucocele apendicular, caso clínico y revisión bibliográfica. Revista Médica de Costa Rica y Centroamérica. 2009; 67: 431-434.

22. Machado NN, Machado NO. Neurogenic appendicopathy: a historical and contemporary review. World J Colorectal Surg. 2014; 4: art. 1.

Correspondencia:

Dr. Sinuhé Álvarez-Álvarez

Dr. Balmis Núm. 142,

Col. Doctores,

Del. Cuauhtémoc, 06720,

Ciudad de México, México.

Tel.: (55) 56427682

E-mail: sinuhealvarez@hotmail.com 